\title{
Cadenas de vulnerabilidad en el sistema de protección a la infancia. El acogimiento familiar fallido como factor de riesgo para el truncamiento adoptivo
}

Chains of vulnerability within the child protection system: failed foster care as a risk factor for adoptive dissolution

Lourdes García Tugas

Profesora Colaboradora, Universitat Oberta de Catalunya (Barcelona, España)

mgarciatu@uoc.edu

Jorge Grau Rebollo

Departamento de Antropología Social y Cultural. Universidad Autónoma de Barcelona.

jordi.grau@uab.es

\section{CRIANZA EN CONTEXTOS DE VULNERABILIDAD SOCIOCULTURAL} MONOGRÁFICO COORDINADO POR JORGE GRAU REBOLLO

\section{RESUMEN}

El acogimiento familiar es una medida de protección a la infancia diseñada para proporcionar un entorno de crianza temporal a menores que se encuentran en una situación de desamparo familiar. Aunque estos emplazamientos suelen ser exitosos y contribuyen a mejorar la situación de vulnerabilidad de los menores acogidos, su posible fracaso puede dificultar una nueva acogida o una eventual adopción futura. En este artículo abordamos la repercusión del acogimiento familiar fallido en el futuro de los menores implicados. Partiendo del examen de documentación oficial de difícil acceso respecto a truncamientos adoptivos, determinamos las características de los casos de acogida familiar en menores adoptados internacionalmente en Cataluña y examinamos las consecuencias que tuvo sobre ellos. Este análisis permite concluir que el acogimiento fallido puede constituir un factor relevante de riesgo para un posible truncamiento en futuros procedimientos de adopción.

\section{ABSTRACT}

Foster care is a child protection measure designed to provide a temporary childrearing environment for children who find themselves in a situation of family neglect. Although these placements are often successful and contribute to improving the fostered children's vulnerable situation, their possible failure may hinder a new foster care placement or an eventual future adoption. In this article, we address the impact of failed foster care on the children's future. Based on the examination of official documentation that is difficult to access regarding adoption truncations, we determine the characteristics of foster care cases in internationally adopted minors in Catalonia and examine the consequences it had on them. This analysis allows us to conclude that failed foster care may constitute a relevant risk factor for a possible truncation in future adoption procedures.

Palabras ClaVe

infancia | vulnerabilidad | protección | acogimiento familiar | truncamiento adoptivo

KEYWORDS

children | vulnerability | protection | foster care | adoptive truncation

\section{Introducción}

Los congresos internacionales sobre "los niños" que se fraguaron desde finales del siglo XIX, posibilitaron la construcción de una "cultura común" (Rollet 2001) con relación a su protección, que acabaría cristalizando en políticas públicas orientadas al bienestar infantil. Los principales focos de discusión giraban en torno a una perspectiva jurídica y social, junto a otra más pragmática y médicohigiénica (Dávila y Naya 2006). Sin embargo, no fue hasta la promulgación de la Declaración Universal de los Derechos Humanos (1948) cuando se marcó un punto de inflexión que sentaría las bases para la posterior Declaración de los Derechos del Niño (1959), donde se definió por vez primera el concepto jurídico de interés superior del menor (que se convertía así en sujeto de derecho).

Posteriormente, la Convención sobre los Derechos del Niño (1989) cosechó el apoyo de la gran mayoría de países y desarrolló la protección a menores en situaciones de vulnerabilidad y bajo cualquier forma de explotación y violencia (Dávila y Naya 2006: 82). Con ello, se mantenía el principio de su interés superior sustanciado en aspectos como la responsabilidad parental en la crianza, la protección de menores 
privados de su medio familiar o la aplicación de medidas como la acogida y la adopción. Desde entonces, los gobiernos han implementado políticas de protección para las niñas, niños y adolescentes (en adelante, NNA), desarrollando legislativamente los principios de la Convención.

En España, la administración pública es el organismo responsable de velar por el adecuado cumplimiento de las disposiciones recogidas en la Ley 26/2015, de 28 de julio, de modificación del sistema de protección a la infancia y a la adolescencia (Ley del Menor). Según dicha ley:

"En situación de riesgo de cualquier índole, la intervención de la administración pública competente deberá garantizar (...) los derechos del menor y se orientará a disminuir los indicadores de riesgo y dificultad que incidan en la situación personal, familiar y social en que se encuentra, y a promover medidas para su protección y preservación del entorno familiar" (BOE 2015: 21).

Así, el acogimiento consituye una medida orientada a garantizar el correcto cuidado y la custodia del menor mediante su ingreso en un centro o establecimiento (acogimiento residencial), o su emplazamiento con una persona o núcleo familiar (acogimiento familiar) que colaboran con la administración en el ejercicio de estas medidas de protección (Observatorio de la infancia 2020). Pese a constituir medidas excepcionales, a 31 de diciembre de 2019 se encontraban bajo su amparo 50.272 menores, de los cuales 42.529 estaban en acogimiento, siendo residencial el $54,5 \%$ y familiar el $45,5 \%$ restante. En lo que a este último se refiere, predomina claramente el que acontece en el seno de la familia extensa $(65 \%$ del total), frente al que recurre a una familia ajena $(35 \%)$. Si examinamos la evolución temporal, se aprecia durante el quinquenio 2015-2019 un aumento espectacular de menores en acogimiento residencial $(70,7 \%)$, mientras que en la variante familiar la tendencia es inversa, apreciándose una reducción del 4,22\%. Una parte de esta considerable desproporción obedece a la incidencia del número de menores extranjeros no acompañados (MENA; también denominados MMNA, menores migrantes no acompañados): solo en 2019 se computan 11.380 menores tutelados, el $99 \%$ de los cuales se encuentra bajo esa medida de protección. No obstante, la fiabilidad del registro de este tipo de migrante menor de edad ha sido puesta en duda en repetidas ocasiones y desde diversas instancias (Martín 2020).

En Cataluña, a 31 de diciembre de 2019 había 5.677 menores en acogida residencial (60\%) y 3.699 en la modalidad familiar (39\%) (Observatorio de la infancia 2020). También aquí la incidencia de los MMNA es considerable: según datos de la Dirección General de Atención a la Infancia y a la Adolescencia (DGAIA), en aquel momento se computaban en Cataluña 3.077 menores de edad no acompañados sin referentes familiares, 120 de los cuales habían llegado durante el último mes (DGAIA 2019: 12).

Sin embargo, no nos ocuparemos aquí de este colectivo, sino que nos centraremos en un fenómeno todavía poco estudiado en España y en Cataluña: el de los acogimientos fallidos y su posible incidencia en un posterior truncamiento adoptivo. Sobre este ámbito, algunas investigaciones precedentes han señalado la escasez de investigaciones que encaren el carácter y frecuencia de ciertos predictores de las rupturas adoptivas y la todavía menor presencia de estudios que se centren en acogimientos fallidos (Sattler and Font 2020), así como en la situación de niños, niñas o adolescentes obligados a reintegrarse en el sistema de acogimiento tras una adopción (Brodzinsky and Smith 2018). En esta línea, Dellor y Freisthler (Dellor and Freisthler 2018) reclaman la necesidad de investigaciones que analicen las rupturas adoptivas, pese a las enormes dificultades legales y administrativas de acceso a los expedientes, y que exploren los factores concretos que pueden incidir en esos truncamientos.

Nuestra investigación contribuye a rellenar este vacío, tras conseguir acceder a la totalidad de fuentes que existían en Cataluña entre 1998 y 2014, además de analizarlas en profundidad y contactar con dos jóvenes que pasaron por este proceso, junto con la información recabada en entrevistas a dos familias de acogida con alguna experiencia fallida. Como explicaremos en el apartado siguiente, la irrupción de la COVID-19 en marzo de 2020 ha condicionado severamente el trabajo de campo con jóvenes extutelados y con familias de acogida, limitando drásticamente las posibilidades de acceso. Con todo, el estudio realizado permite continuar y ampliar investigaciones de referencia sobre adopciones truncadas en España como los de Berástegui (2003), Paniagua y otros (2018) o Paniagua y Palacios (2016).

\section{Método}

\subsection{Procedimiento de investigación}


Nuestra investigación se basa en tres ejes principales:

1) Revisión de literatura académica relevante sobre medidas de acogimiento.

2) Análisis de la información correspondiente a 112 expedientes oficiales, 59 recogidos por la DGAIA y 53 por el Instituto Catalán del Acogimiento y la Adopción (ICAA).

3) Entrevistas en profundidad a familias adoptivas y a jóvenes (ya mayores de edad) que hubiesen pasado por un proceso de truncamiento adoptivo, debiendo ser reintegrados al mecanismo institucional de tutela. (Por razones de coherencia metodológica y narrativa, estos testimonios forman parte de la investigación, pero no se han incluido en los casos abordados en los apartados 4.2 y 5 ).

El periodo total de investigación comprende entre 2014 y 2020, aunque ha debido ejecutarse a diferente ritmo según el eje abordado. Mientras que la revisión y actualización de literatura ha podido llevarse a cabo de forma ininterrumpida durante todo el periodo, el acceso a los expedientes fue un proceso mucho más complejo. Como bien señalan Dellor y Freisthler (2018), aproximarse a las adopciones fallidas comporta una considerable dificultad debido a la confidencialidad que la ley establece al respecto, lo que hace sumamente complicado acceder a documentación oficial. En nuestro estudio, tras meses de solicitudes y trámites con la administración pública competente, conseguimos disponer de la totalidad de expedientes emitidos entre 1998 y 2014.

Igualmente complejo resulta acceder a familias con casos fallidos de acogimiento y tanto o más complicado ha sido poder localizar y entrevistar a jóvenes (ya mayores de edad) con experiencias previas de truncamiento y reinstitucionalización. A los mecanismos que la ley establece para garantizar su anonimato, Eldridge, John y Gleeson (2020) añaden el peso del estigma y los prejuicios negativos como grandes desincentivadores de confianza, lo que reduce considerablemente el espectro de posibles participantes. Cabe añadir que, en un ámbito tan sensible como el que aboradamos, la eclosión de la pandemia agravó esta desincentivación, llegando a dificultar extraordinariamente el contacto con familias y jóvenes extutelados. Algunas de las familias acogedoras rechazaron la propuesta alegando motivos de confidencialidad y otras declinaron al encontrarse en conflicto con la administración (incluso en algún caso, por consejo legal, la familia decidió interrumpir las entrevistas no presenciales). Además, la propia administración modificó radicalmente sus prioridades de atención, cancelando reuniones y entrevistas ya concertadas y reorientando sus líneas de actuación. Pese a todo, hemos podido trabajar con 62 familias adoptivas, 2 jóvenes extutelados tras una adopción truncada y 27 casos de acogimiento truncados.

\subsection{Definición de conceptos}

No es fácil construir una definición unificada del término "acogimiento" desde una perspectiva transnacional, debido a que un mismo término adquiere distintos significados en diferentes zonas geográficas (1), teniendo en cuenta: a) si el menor es "acogido" por familia extensa o ajena, b) si se trata de una medida institucional o informal, c) la duración del "acogimiento" o d) la noción del tipo de "familia" acogedora (Colton y Williams 1997; los autores ponen el ejemplo de Israel y los cluster, donde hasta veinte familias de acogida pueden vivir en un mismo barrio y acoger a varios niños cada uno).

En España, todas las medidas de protección se determinan desde las instituciones públicas competentes de cada Comunidad Autónoma sobre el principio del interés superior del menor y suponen, en primera instancia, una actuación admnistrativa. Cuando se inicia el proceso judicial, la supervisión descansa en el ministerio fiscal y la activación, progreso y conclusión de cada procedimiento dependen de decisiones judiciales. Esta supervisión se activa en contextos de desamparo, entendiendo como tal: "[la] situación caracterizada por la no existencia de la cobertura de ciertas necesidades básicas para el correcto desarrollo de un menor" (Real y otros 2020: 8).

Conforme a la definición adoptada por el Observatorio de la Infancia, entenderemos aquí por acogimiento familiar:

"[La] medida de protección de niñas, niños y adolescentes que, con carácter administrativo o judicial, otorga la guarda de una niña, niño o adolescente a una persona o núcleo familiar con la obligación de velar por él, tenerlo en su compañía, atenderlo, alimentarlo, cuidarlo y procurarle una formación integral a fin de proporcionarle una vida familiar sustitutiva o complementaria de la propia" (2020: 17). 
Esta medida puede adoptarse con carácter de urgencia (aplicable a menores de seis años, por un plazo inferior a seis meses), temporal (durante un periodo máximo de dos años, prorrogables en función de la situación del menor) o permanente (tras concluir el periodo de dos años, o bajo determinados supuestos como la imposibiliad de reintegración familiar o la existencia de necesidades especiales por parte del menor).

Por el contrario, el acogimiento residencial refiere al "cuidado y custodia del menor de edad (...) cuando se lleva a efecto mediante el ingreso del menor de edad en un centro o establecimiento, sea propio o colaborador" (Observatorio de la infancia 2020: 16).

Por truncamiento adoptivo entenderemos la disolución de una adopción plena legalmente constituida, que comporta la separación definitiva de un menor de su familia adoptante, retornando la custodia a la administración pública competente. No hablamos, pues, aquí de adopciones que no llegan a completarse, sino de procedimientos legales que han generado una nueva filiación con pleno efecto jurídico (Berástegui 2018; Brodzinsky y Smith 2018).

Además, como apuntan diversos estudios precedentes, este truncamiento y posterior reintegración establece una categoría diferente de menor tutelado (Kim 2017, Rolock 2015) y requiere más investigación orientada específicamente a los estresores y factores de riesgo asociados a este proceso (Dellor y Freisthler 2018, Sattler y Font 2020). Este carácter singular se acentúa cuando el perfil de menor responde al de un niño o niña que ha pasado por un proceso de adopción internacional, puesto que a los estresores anteriormente mencionados se suman las potenciales percepciones sociales derivadas de su fenotipo o país de origen. Las singularidades que, a este respecto, conlleva la adopción internacional han sido ya profusamente abordadas en numerosos estudios (Grotevant 2000, Howell 2009, Marre y Briggs 2009 o Palacios y Brodzinsky 2010, entre otros).

\section{El acogimiento familiar como medida de protección a la infancia: éxitos y fracasos}

\subsection{El acogimiento familiar en el conjunto del sistema de protección}

Cualquier medida de protección a la infancia constituye un mecanismo excepcional, puesto que se activa únicamente cuando un menor se encuentra en riesgo de desamparo. A esta situación pueden conducir diversas causas: desde malos tratos hasta la inadaptación familiar tras un proceso de adopción. Pese a la práctica imposibilidad de tener registros fiables sobre el número de NNA privados de su familia de origen, Robert McCall calculó que a mediados de la primera década de los 2000 , considerando un total de 93 países, habría alrededor de 143 millones, de los cuales solo 8 millones estarían institucionalizados (2011: 258).

En España, el porcentaje de menores en el sistema público de protección puede considerarse muy bajo si lo comparamos con el total de jóvenes por debajo de los 18 años: a 31 de diciembre de 2019 había en España, según datos oficiales del INE, 7.826.403 NNA comprendidos entre los 0 y los 17 años, de los cuales solo el $0,6 \%$ se encontraba bajo un mecanismo de protección (50.272). Sin embargo, no debe perderse de vista lo siguiente:

"Más allá de los números y de los factores, cada fracaso es la historia de un niño o niña al que no hemos podido asegurar la vida familiar a la que tenía derecho, la historia de un niño o niña que, en muchas ocasiones, ya no tendrá una nueva oportunidad en familia. El fracaso también es la historia de una familia que soñó en un proyecto familiar abierto a un niño al que no pudo o no supo cómo convertir en hijo, cómo cuidar o cómo proteger. Cada ruptura, es finalmente, el fracaso de un sistema cuya única motivación es proteger a los niños pero que, en muchas ocasiones, no acierta en el modo de hacerlo con éxito" (Berástegui 2018: 21).

López y otros (2014) recuerdan que estos mecanismos de protección no pueden entenderse adecuadamente sin tener en cuenta las características sociales y culturales del país en el que se implementan, y en el caso español se combina una fuente tradición familista con un tejido institucional débil de apoyo a la familia que, en parte, explican la relativamente tardía consolidación del acogimiento familiar:

"Los países mediterráneos, particularmente los de fuerte influencia de la Iglesia católica, han 
mantenido el predominio de las instituciones de acogida de menores desprotegidos (...). En España, el acogimiento familiar se introduce en el marco jurídico en 1987 (...) y todavía en la actualidad presenta cifras que indican un lento y costoso desarrollo" (López y otros 2014: 286).

Este tránsito de la beneficencia a la protección pública obligó a desarrollar un cuerpo jurídico específico (2). que ha mantenido en todo momento el carácter subsidiario y excepcional del acogimiento: las instituciones competentes podrán delegar la crianza de un menor en familias sustitutas únicamente cuando lo reclame su interés superior. Sin embargo, frente a otros países con mayor tradición jurídica en este ámbito, el acogimiento familiar en España aparece caracterizada en ocasiones como "una de las grandes asignaturas pendientes de nuestra proteccioón infantil" (Fernández del Valle y otros 2008: 34), lo que también se tradujo en una atención académica más tardía (para una revisión de algunas investigaciones pioneras de referencia en España, véase Fernández del Valle y otros 2008). Un factor adicional a tener en cuenta es la variación que pueden ofrecer entre sí las diferentes comunidades autónomas, por cuanto cada una tiene competencia para aprobar y desarrollar leyes reguladoras propias. Así, en Cataluña, se desarrolló un reglamento de protección de menores desamparados y de la adopción mediante la promulgación del decreto 2/1997, de 7 de enero, o más recientemente la Ley 14/2010, de 27 de mayo, de los derechos y las oportunidades en la infancia y la adolescencia.

\subsection{Cuando el mecanismo falla: resquicios de vulnerabilidad sobrevenida en los sistemas de protección}

Sin embargo, en ocasiones los mecanismos de protección terminan amplificando la condición vulnerable del menor al no conseguir resolver sus necesidades asistenciales mediante la activación de las garantías judiciales, lo que prolonga la cadena de vulnerabilidad en la que se ve inmerso. Pese a la atención que el acogimiento ha despertado en los últimos años, Real y otros (2020: 19) constatan la escasez de estudios que profundicen en las variables que pueden determinar su éxito o fracaso, centrándose la mayor parte de veces en aspectos relativos a los déficits de previsión del sistema social ante la mayoría de edad de los menores en protección, las motivaciones de las familias acogedoras, los estilos educativos o la información recibida durante el proceso de acogimiento.

De las investigaciones que sí han abordado aspectos problemáticos del acogimiento y de la adopción, podemos destacar lo siguiente:

a) Los riesgos emocionales y psicológicos, bien glosados en su momento por Wolf (2008) y Palacios y Brodzinsky (2010) y uno de cuyos exponentes más recientes es la aproximación al desarrollo de la confianza en otros por parte de menores que han pasado por servicios de acogida (Eldridge y otros 2020).

b) La influencia del deterioro del bienestar y de las experiencias insatisfactorias de acogimiento sobre la vida posterior del menor (Meakings y Selwyn 2016, Brännström y otros 2020).

c) Los factores que pueden asociarse a aspectos identitarios (Colbridge y otros 2017) o al desarrollo de mecanismos efectivos de apego relacionados con la familia acogedora, con el emplazamiento en sí o con los propios menores (West y otros 2020).

d) Los estudios comparativos entre acogimientos familiares y residenciales y su resultados sobre las conductas y percepciones futuras de los menores implicados ( $\mathrm{Li}$, y otros 2017).

e) Los procesos de reinstitucionalización o reemplazamiento familiar (Rolock 2015, Rolock y otros 2018).

f) Las limitaciones institucionales en el apoyo a familias acogedoras (Twigg y Swan 2007) y la saturación en las cargas laborales de profesionales relacionados con los servicios sociales (Schwartz 2008).

Cabe señalar, no obstante, que no todas las estrategias de protección pasan por procedimientos formales de emplazamiento. En ocasiones, pueden encontrarse mecanismos de transferencia informal de responsabilidades de crianza de un/a niño/a bajo la forma de una circulación intencional y consentida (Leinaweaver 2008, Grau 2011). Sin embargo, no debe confundirse esta movilidad, orientada a asegurar el bienestar del menor, con conductas no comunicadas a los servicios sociales o realizadas sin requerir el consentimiento de la autoridad judicial en el marco de procesos adoptivos que no están funcionando y donde, por ejemplo, los padres adoptantes pueden optar por emplazar a su hijo/a en centros residenciales privados (Brodzinsky y Smith 2018). Otro error frecuente consiste en asimilar estas 
prácticas informales a la explotación infantil o al tráfico de menores (Howard 2011, Chanyalew y Abebe 2016).

\subsection{Estrategias informales de crianza y protección}

En el ámbito internacional, podemos apreciar la extensión de prácticas de circulación infantil que, con carácter informal, han proporcionado al menor un entorno alternativo de protección. Algunas de estas formulas las hallamos descritas en Goody y Goody 1967, Goody 1969, Brady 1976, Fonseca 2002 y 2006b, Leinaweaver 2007 y 2008, o Grau 2011, mostrando cómo el emplazamiento de hijos e hijas en otros núcleos familiares deviene una estrategia de superación de situaciones que constriñen, temporal o definitivamente, la crianza del menor en su familia de origen. Desde esta perspectiva, la circulación infantil es un recurso táctico de crianza ( $\mathrm{y}$, a menudo, de cohesión social) que resigue patrones sociales de apoyo y reciprocidad cuyas lógicas culturales rebasan las costuras de los sistemas jurídicos, tanto a nivel local como internacional. Así, por ejemplo, Fonseca (2004) relata cómo en Brasil no era infrecuente que mujeres de familias que atravesaban situaciones especialmente precarias emplazaran temporalmente a algunos/as de sus hijos/as en otros hogares familiares, hasta que la situación mejorase y pudiesen reclamarlos.

Así, como sugiere Leinaweaver (2010), la circulación infantil constituye un recurso de afrontamiento ante la vulnerabilidad económica. A lo largo de nuestra investigación hemos encontrado diversos casos en los cuales la historia del menor adoptado internacionalmente presenta uno o más emplazamientos informales de esta naturaleza. Por ejemplo, en los expedientes de menores bolivianos hemos detectado menores que fueron adoptados internacionalmente y que habían sido reubicados previamente en diferentes hogares. En un caso, una madre dejó a su hijo en una tienda y pidió a la propietaria que se hiciera cargo de él un momento, aunque nunca más regresó. Esta propietaria asumió la crianza del niño durante años, hasta que se vio obligada a cerrar su negocio y el deterioro de su situación económica derivó en un nuevo emplazamiento del menor. Esta situación se repitió hasta que las autoridades detectaron el caso y medió una declaración de desamparo.

También en Filipinas hallamos las dos fórmulas de acogimiento. Sin embargo, en este país la administración ofrece a las familias que provisionalmente no pueden hacerse cargo de sus hijos e hijas la posibilidad de ingresarlos en un orfanato, donde se les cuida hasta el regreso de los progenitores $u$ otros familiares, asimilándose esta vía a la de una institucionalización temporal. En China, hemos hallado casos en los cuales las propias cuidadoras del orfanato se hicieron cargo de alguna niña, llevándosela a su hogar tras la jornada laboral. Algunas familias adoptantes han recibido cartas y mensajes de familias de acogida interesándose por la niña adoptada (e incluso una pareja relataba cómo toda la familia extensa de acogida se había presentado en el hotel para despedirse de ella).

\section{Conexiones entre las adopciones truncadas y el acogimiento familiar fallido}

\subsection{Estudios precedentes sobre fracaso adoptivo}

Dentro del ámbito académico de las adopciones internacionales existen diversos estudios que se han aproximado a los fracasos adoptivos. A comienzos de los años ochenta Zwimpfer (1983) llevó a cabo un estudio con el fin de determinar los indicadores que podrían actuar como predictores del riesgo de fracaso. Años después, Festinger (2002: 517), tras constatar la dificultad de acceder a registros fiables respecto al número de fracasos adoptivos, advierte sobre el problema que supone cada nuevo reingreso de un menor en el sistema de acogida, al ser considerado un caso nuevo, sin establecer un vínculo adecuado con la situación precedente. Pese a todo, constata una tasa muy baja de rupturas en comparación con el conjunto de adopciones, extremo que corroborarán diversos estudios posteriores en diferentes países.

Depurar posibles predictores de truncamiento se convertirá en un leitmotiv recurrente entre estudios sobre este fenómeno, junto con la constatación de la escasez de investigaciones, tanto a nivel internacional como, particularmente, en España (Berástegui 2003 y 2018, Paniagua y Palacios 2016, Dellor y Freisthler 2018, Paniagua y otros 2018, Sattler y Font 2020).

Por otro lado, la carga semántica del término "truncamiento" (dissolution, en su acepción inglesa más frecuente) dio lugar a propuestas terminológicas que pretendían acuñar expresiones menos negativas. 
Así, Festinger y Maza (2009) propondrán hablar de emplazamientos post-adoptivos (post-adoption placement), lo que encajaría mejor con las eventuales adopciones subsiguientes, en caso de poder llevarse a término. Otras líneas de trabajo se han centrado en los procedimientos que responden a necesidades especiales por parte del menor (Kim 2017), en trayectorias de emplazamiento o en la etnicidad como variables relevantes en estas disoluciones (Kim y otros 2019), además del papel que pueden desempeñar factores como el género, los malos tratos o la exposición al consumo de drogas (Dellor y Freisthler 2018).

En España, constituyen una referencia obligada los trabajos de Berástegui (2003 y 2018, entre otros), Palacios, Sánchez-Sandoval y León (2009), Paniagua y Palacios (2016) o Paniagua y otros (2018), quienes coinciden en señalar la importancia de una mejor formación y de un acompañamiento sostenido de las familias adoptivas, junto con la necesidad de desarrollar mecanismos más ágiles de detección precoz y de mantener un registro actualizado y sistemático de adopciones truncadas.

\subsection{La situación en Cataluña}

\subsubsection{Cuestiones legales y tipos de acogimiento familiar}

En Cataluña las políticas sociales de protección a la infancia se implementaron en 1980, de la mano de las transferencias competenciales desde el gobierno central (Casas 2012).

A partir de ese año se desarrolló un proceso legislativo en materia de infancia que culminó con la actual Ley Orgánica 14/2010, de 27 de mayo, de Derechos y Oportunidades en la Infancia y Adolescencia (LDOIA) que, junto a la Ley 25/2010, de 29 de julio, del libro segundo del Código Civil de Cataluña, supusieron el fin de la dispersión normativa (Allueva 2011: 4). La LDOIA define la medidas de protección pública y los recursos necesarios para implementarlas y asienta el sistema de protección de los/as NNA en situación de riesgo y desamparo. Adicionalmente, establece los supuestos que constituyen situaciones de riesgo (art. 102.2) y prioriza la colaboración de los progenitores o tutores con los servicios sociales. Sin embargo, cuando esto no sea posible, la Ley prevé la incoación de un procedimiento de desamparo. En su artículo 105.1, establece lo siguiente:

"Se consideran desamparados los niños o los adolescentes que se encuentran en una situación de hecho en la que les faltan los elementos básicos para el desarrollo integral de la personalidad, siempre que para su protección efectiva sea necesario aplicar una medida que implique la separación del núcleo familiar" (Generalitat de Catalunya 2010).

De este modo, la declaración de desamparo requiere de una resolución administrativa que en Cataluña queda en manos de la DGAIA, que asume así funciones tutelares. Entre la activación de estas medidas, se reconocen seis tipos de acogidas en función de las necesidades de los menores y de la situación de la familia:

- Acogimiento de urgencia y diagnóstico: permite la estancia con una familia mientras se analizan las circunstancias que han aconsejado la separación de la familia de origen. Está dirigido a niños/as de hasta 6 años y su duración comprenderá el tiempo necesario para la realización del estudio.

- Acogimiento de corta duración: el emplazamiento temporal con una familia prevé la reintegración al entorno de origen antes de dos años.

- Acogimiento de larga duración: el emplazamiento familiar se prolonga hasta que se da por superada la circunstancia que ha motivado la separación de la familia de origen.

- Acogimiento de fines de semana y vacaciones: dirigido a NNA mayores de nueve años que se encuentran ingresados en centros residenciales o que precisan un ambiente familiar. El/la menor convive con la familia colaboradora los fines de semana y las vacaciones escolares durante el tiempo que se considere beneficioso para él/ella.

- Acogimiento en unidades convivenciales de acción educativa: dirigido a NNA tutelados con necesidades educativas especiales, enfermedades crónicas y/o trastornos de la conducta, y grupos de hermanos/as que requieran una atención más personalizada. Alguno de los miembros del entorno de acogida debe poseer titulación, formación y experiencia suficiente para atender las necesidades especiales de los menores. 
- Acogimiento permanente: se acuerda ante la previsión de un desamparo definitivo, siempre y cuando no se considere más favorable un acogimiento preadoptivo (o este no sea posible).

- Acogimiento preadoptivo: la finalidad última es la adopción del/la menor.

\subsubsection{Perfiles de NNA}

Una de las primeras constataciones de nuestra investigación fue la escasa información disponible acerca de los antecedentes de menores afectados por situaciones de truncamientos adoptivos (García Tugas 2019). Es, por tanto, probable que otros niños y niñas que aparecen en los expedientes también hayan tenido experiencias de acogimiento o circulación en su país de origen que no han quedado registradas y, por consiguiente, no pueden ser analizadas en la actualidad.

Tras el examen de toda la información administrativa disponible, apreciamos que 72 menores adoptados internacionalmente en Cataluña habían sido nuevamente desamparados. Tanto los orígenes como las experiencias de estos niños y niñas con relación al acogimiento son diversos. De ellos, 27 (37\%) tuvieron como mínimo una experiencia de acogida antes de la adopción y/o con posterioridad a esta, diferenciándose tres supuestos:

a) Experiencias de acogida, previas a la adopción, únicamente en el país de origen (8 niños y niñas).

b) Acogimientos en familias tras fracasar la adopción (14 niños y niñas).

c) Combinación de experiencias de acogida (5 niños y niñas), tanto en el país de origen, antes de ser adoptados/as, como en el de destino, después de la adopción.

El truncamiento de la adopción vino motivado, en estos 27 casos, por la renuncia de los adoptantes (59\%), las denuncias de la situación de riesgo del menor (37\%) o la demanda del propio menor (11\%). El porcentaje total supera el $100 \%$ porque en algunos casos hubo más de una motivación.

\section{A) Niñas y niños emplazados únicamente en el país de origen}

De los expedientes examinados, la distibución por género es similar: el $49 \%$ eran niñas y el $51 \%$ niños. Respecto a la edad de los menores emplazados en origen, en el momento de ser adoptados el rango oscila entre los 3 y los 10 años, sin que predomine ninguna franja específica. En todos los casos, fueron desplazados a otros entornos familiares en su país de origen, resultando el $58 \%$ en emplazamientos informales y casi el $42 \%$ en acogimientos regulados legalmente. Por países, en Colombia y Rusia el acogimiento temporal se da formalizadamente en familia ajena, mientras que en Etiopía y Perú encontramos básicamente acogimiento informal en familia extensa, situación también detectada en Ucrania y Brasil. Hungría y Bulgaría son países con predominio de acogida preadoptiva.

Si examinamos las causas que derivaron en el acogimiento (véase el gráfico), podemos apreciar un cierto predominio del abandono por parte de los progenitores, seguido del fallecimiento de los mismos y del reacogimiento de menores tras fracasar el primer emplazamiento (fuese formal o informal), debido a la negligencia y a los malos tratos durante la crianza. A mayor distancia encontramos la renuncia y la retirada de la patria potestad como medidas legales interpuestas por las autoridades, y los emplazamientos informales (circulación de niños/as) consensuados entre progenitores y acogedores, sin que se consigne de modo concluyente la motivación. En todos estos casos, los expedientes refieren a situaciones vulnerabilidad económica y social, así como al consumo de alcohol y/o de drogas. 


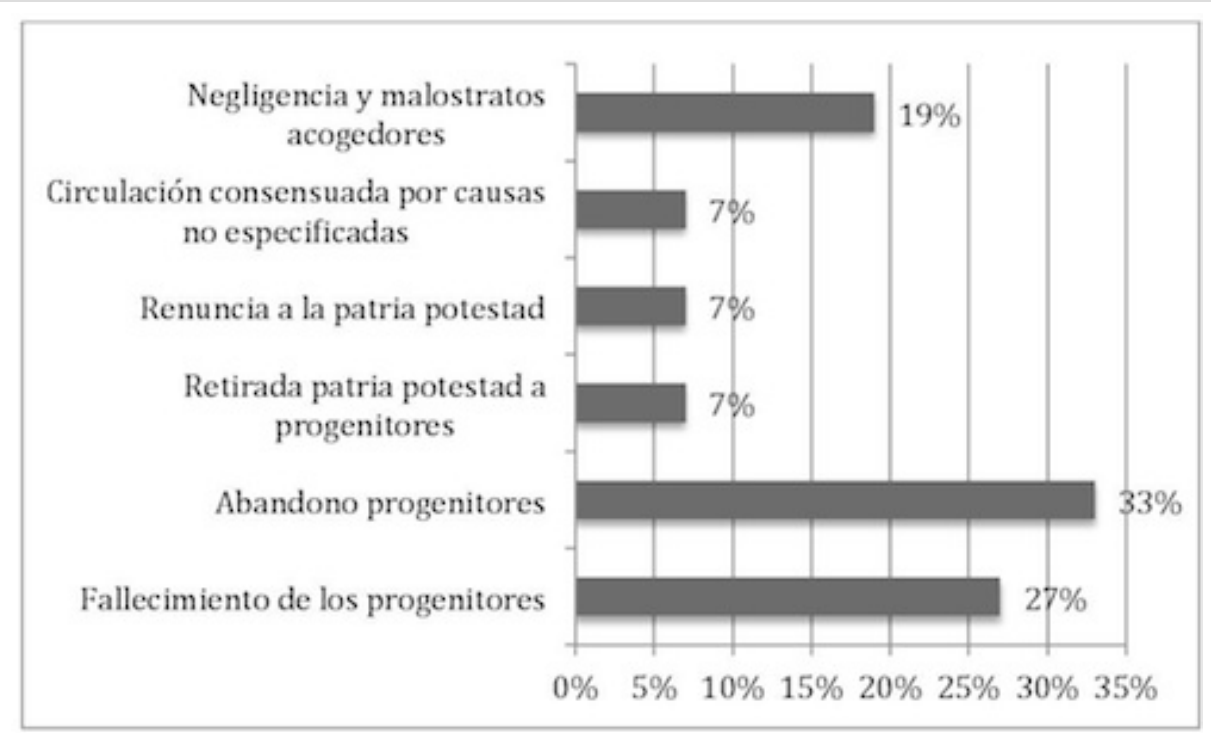

Gráfico: causas de la circulación o del acogimiento (elaboración propia)

Atendiendo al país de origen, en Ucrania, Hungría y Colombia el motivo principal fue el abandono por parte de los progenitores. Por otro lado, en Perú y en Etiopía el fallecimiento de los mismos es la causa mayoritaria que propicia el acogimiento o emplazamiento informal, mientras que la retirada de la patria potestad prácticamente se circunscribe a Rusia y la renuncia a Bulgaria. En sintonía con lo apuntado por Fonseca (2004 y 2006a), hemos detectado situaciones de acogimiento informal (circulación de niños/as) en Brasil, aunque no se recogen las causas que motivaron tal medida. También se aprecia esta circulación previa a la adopción internacional en Perú, en línea con lo apuntado por Leinaweaver (2007), si bien en este caso sí se especifica que el emplazamiento se suspendió tras detectar negligencia grave en la crianza del menor.

\section{B) Niñas y niños con acogimiento postadoptivo}

De los 27 niños y niñas que han pasado por una experiencia adoptiva, diecinueve pudieron ser acogidos después del fracaso adoptivo. De estos, el $42 \%$ lo fue más de una vez, al fracasar el primer acogimiento tras la adopción. A excepción de un caso, todos los acogimientos fueron formales: uno en familia extensa y el resto tutelados por la administración. El acogimiento informal se circunscribe al caso de dos menores que habían sido "adoptados" de forma irregular. Ante las dificultades relacionales que los "adoptantes" experimentaron con uno de ellos, optaron por delegar su crianza en una familia distinta, que posteriormente acogió legalmente a los menores hasta su mayoría de edad, momento en que se cerró el expediente.

Cabe destacar que los acogimientos por parte de la familia extensa se debieron a casos en los cuales la administración valoró que los NNA se hallaban en situación de riesgo con sus padres adoptivos. Para garantizar su bienestar y protección, dos de ellos fueron temporalmente acogidos por un familiar hasta que los adoptantes recuperaron su tutela. En otro caso, dos hermanas (una en desamparo por la renuncia de los adoptantes y la otra tutelada por la administración) fueron acogidas por dos nucleos familiares distintos pertenecientes a la familia extensa de los adoptantes, quienes, ante la situación de malos tratos que habían vivido las niñas, decidieron romper su relación con estos. El primer emplazamiento de una de las niñas no fructificó y fue acogida por dos familias sucesivamente; así, las dos hermanas se vieron obligadas a vivir separadas, aunque pudieron mantener la relación entre sí.

En otro de los casos examinados, los adoptantes quisieron renunciar a la adopción cuando aun se encontraban en el país de origen del menor. Como esto no fue posible, optaron por esperar a su llegada a Cataluña para formalizar la renuncia ante el ICAA. El menor, de dos años y medio, fue entonces acogido por una familia que, posteriormente, lo adoptó.

Un caso especialmente traumático es el de dos hermanos gemelos (niño y niña) adoptados por una pareja. A causa de las dificultades conductuales de la niña, los adoptantes manifestaron su voluntad de renunciar a su adopción, aunque decidieron seguir adelante con la de su hermano; sin embargo, desistieron cuando la administración no estimó la separación y la consiguiente declaración de desamparo 
otorgó la guarda provisional de ambos menores a una familia acogedora. Un año después, quisieron recuperar la tutela de los niños e iniciaron un proceso judicial de oposición a la medida interpuesta por la administración pública, pero la sentencia falló a favor de la administración, de modo que los menores pudieron permanecer con la familia de acogida que estaba tramitando ya su adopción. Seis años más tarde, la madre adoptiva se personó varias veces en la escuela con fotos de los niños (tomadas cuando aun estaban en su país de origen) y se las enseñó con la promesa de entregárselas si iban a su casa. Además, les pidió que no lo explicaran a nadie o a ella ingresaría en prisión. La familia acogedora achacó los vaivenes en la conducta de la niña durante ese periodo a la entrada en la preadolescencia, hasta que les reveló la situación y pudieron poner el caso en conocimiento de la administración.

Otras veces, en cambio, los acogimientos no fructificaron y en ocasiones el menor debió permanecer en una institución hasta su mayoría de edad (pasando a residir en pisos de extutelados tras cumplir los 18 años). De los cinco casos examinados en esta situación, el fracaso parece deberse a dos grandes factores:

1) Los efectos psicológicos y emocionales de la historia de vida del menor. Fue el caso, por ejemplo, de una joven acogida por dos familias con quien estableció una buena relación, pero sin poder sostener la convivencia, o de una joven acogida quien, después de siete meses, comunicó la renuncia. En el informe de esta útlima se recoge lo siguiente:

"La familia preadoptiva se ve incapaz de sostener el comportamiento y la manera de relacionarse de la niña, a menudo desafiante y provocadora, con oposición constante a las normas de convivencia familiar y rechazando manifiestamente la figura del padre acogedor. A pesar de ser una familia con recursos educativos y afectivos, ha llegado un punto límite de agotamiento y bloqueo, creándose una dinámica negativista y deterioro de la relación creciente, que no les permite avanzar y que se hace extensiva a todos los miembros que conviven en el domicilio".

2) La presión de los adoptantes sobre los menores en contra de los acogedores. Hallamos tres casos con estas características. En dos de ellos (hermanos), la madre adoptiva apelaba a la "fidelidad" que los niños debían mostrar hacia ella. Según consta en el informe:

"tampoco acepta la integración de los hijos con las familias colaboradoras y no permite que se puede hablar de las vivencias de lo niños con estas. Se valora que las llamadas de la madre estan resultando demasiado perjudiciales. Da mensajes culpabilizadores, sobre todo a la niña, respecto a su convivencia con la familia colaboradora y esto está perjudicando su adaptación (...) finalmente consigue su propósito (...) al verbalizar la niña su malestar y el temor de defraudar a su madre".

Otro joven de 16 años, víctima de maltrato físico y psicológico por parte de sus padres adoptivos, fue acogido en una familia que finalmente renunció al acogimiento:

"Hace tres días que (...) no está con nosotros y ahora, desde la seneridad, quiero describir los últimos acontecimientos para poder recordar y si hace falta, dejar testimonio de cómo (...) ha sido víctima de una autentica "paliza" psicológica brutal desde hacer aproximadamente un mes por parte de la su familia adoptiva. Hace siete meses que (...) vive con nosotros y podemos decir que no ha sido hasta ahora (...) que (...) no ha tenido unos cambios de actitud y carácter considerables".

Pese al fracaso en el proceso adoptivo, estos casos ponen de relieve que la culminación de un acogimiento exitoso depende de la conjugación tres factores esenciales: a) la historia de vida del NNA, el daño que ha sufrido y su capacidad de resiliencia; b) las competencias parentales de los/as acogedores/as; y c) un acompañamiento profesional al menor y a la familia acogedora durante el proceso de acoplamiento.

\section{C) NNA con acogida tanto en el país de origen como en destino, tras su adopción}

Cinco han sido los NNA que fueron acogidos antes de la adopción y después del fracaso adoptivo, procedentes de Etopía, Perú, Ucrania (dos casos) y Rusia. El menor de Etiopía fue acogido por su propia familia extensa debido al fallecimiento de los progenitores, siendo posteriormente entregado a un orfanato. Fue adoptado con 4 años y pasó por dos procesos sucesivos de acogimiento, culminando el último en adopción.

La menor de Perú fue acogida por unos tíos cuando murieron los progenitores. Debido al maltrato 
recibido, les fue retirada la tutela y se determinó su ingreso en un orfanato, desde donde fue adoptada por vía internacional con 9 años. Sin embargo, padeció también malos tratos por parte de la familia adoptiva, lo que desembocó en un acogimiento provisional por una familia y finalmente pasó a convivir fines de semana y vacaciones con otra familia colaboradora.

En uno de los casos de origen ucraniano, la niña fue acogida temporalmente por la familia de su madre e ingresó en un CRAE (Centro Residencial de Acción Educativa) tras el truncamiento adoptivo. Posteriormente, fue acogida hasta su mayoría de edad por la madre de su pareja afectiva.

Finalmente, la niña de origen ruso convivió con una familia acogedora tras la renuncia de la madre biológica. Fue adoptada con 7 años, pero la adopción se truncó solo doce meses después, debiendo ser ingresada en un CRAE. Un tiempo después fue acogida por una familia, pero acabó regresando al CRAE tras evidenciar problemas de conducta. Tras un nuevo intento fallido de acogimiento, volvió al centro, donde tuvo un intento de autolisis.

En todos estos casos la experiencia del NNA con las familias acogedoras de origen es un factor a tener en cuenta con relación al acoplamiento del/la menor con familias de acogida después del truncamiento adoptivo: son menores que sufren el abandono de los progenitores, el intento frustrado de una acogida y el fracaso de una adopción, y cada mala experiencia condiciona más, si cabe, el establecimiento posterior de vínculos de confianza.

\section{Factores de riesgo detectados}

Dos de los principales factores de riesgo que hemos detectado en nuestro estudio son: a) el tiempo que transcurre desde el momento en que la familia biológica renuncia a la tutela del NNA hasta su adopción internacional, y b) la pérdida reiterada de personas referentes para el/la niño/a (sobre todo al condensarse en un corto periodo de tiempo). Por ejemplo, un menor etíope de 3 años de edad, se enfrentó al fallecimiento, primero del padre y después de la madre con una diferencia de tan solo dos años. En ese momento, una tía (no consta si paterna o materna) se hizo cargo de él durante un año entero, tras el cual pasó a servicios sociales y estos lo derivaron al orfanato. La propuesta de asignación se realizó una semana después del ingreso en el centro y el encuentro con los adoptantes tuvo lugar un mes y medio después del ingreso. Tres meses después de formalizar la adopción, los adoptantes alegaron que el niño tenía una "actitud agresiva, con problemas de conducta y mal comportamiento" (transcripción literal del expediente), por lo que decidieron renunciar.

Otro ejemplo lo hallamos en una niña de Perú cuya madre murió cuando ella tenía 4 meses y el padre la dejó a cargo de un tío materno. Cuando una vecina vio cardenales en la espalda de la niña, lo denunció a las autoridades, quienes la emplazaron con otros familiares, que volvieron a maltratarla. Tras este segundo episodio de violencia, se declaró un nuevo desamparo y tres meses después la menor fue adoptada por vía internacional.

En esta línea, otro factor de riesgo es la violencia o abuso sobre estos NNA cuando se encuentran bajo la supuesta protección de familias acogedoras, como hemos visto en estos dos ejemplos relatados. Especialmente perniciosa es la reiteración de malos tratos en sucesivos acogimientos familiares. Es el caso, por ejemplo, de Marta, una niña que, con dos años de edad, perdió a sus progenitores y permaneció cuatro años bajo el cuidado de unos tíos maternos. En aquel momento, estos delegaron informalmente su cuidado a una "media hermana" de la niña, con la que estuvo un año más y que acabo dejando en un hospital al no poder hacerse cargo de ella por motivos económicos. La declaración judicial de desamparo no se produjo hasta dos años después, con lo que ingresó en una Aldea Infantil y fue emplazada con una "mama sustituta" y "hermanos sustitutos" ya con 9 años. Un tiempo después, la niña relató los malos tratos que había recibido tanto por parte de los tíos como de la "media" hermana; además, uno de los "hermanos": "ha llegado a molestar a la niña con actos bastante fuertes [sic], llegando incluso a amenazarla con pegarla si es que comunica de sus insinuaciones" (Fuente: Informe social emitido por la Aldea infantil). Este mismo organismo destacaba que la niña reaccionó ante las situaciones de abandono con depresión y dependencia afectiva hacia terceras personas. Tras permanecer dos años en esta situación, fue adoptada internacionalmente, pero su padre adoptivo abandonó el hogar poco tiempo después y la madre adoptiva, quien mostraba lo que después se describió como signos evidentes de inestabilidad emocional, la maltrató gravemente. 
Más complejo aun es el caso de Jacinto, a quien adoptaron con 9 años y procedente de un país sudamericano (omitimos el país para preservar la confidencialidad de las personas implicadas, dada la abundante información de contexto proporcionada). El informe social que emitieron las autoridades en origen consignaba que el niño era hijo de padre desconocido y sobre la madre se detallaba textualmente que "se trasladó a [provincia] todavía pequeña bajo la responsabilidad de hecho de la Sra. X. No tenía certificado de nacimiento ni conocimiento de sus familiares biológicos. Fue "criada" por la Sra. X hasta la fase de la adolescencia, nunca frecuentó la escuela y por lo tanto no es alfabetizada. Dejó de vivir con la Sra. X porque le pegaba mucho el compañero de ella" (extracto literal del informe).

Cuando nació su hijo, lo dejó bajo los cuidados de la Sra. X, quien "por estar siempre bebida no atendía a las necesidades del menor. El Consejo tutelar retiró al niño de los cuidados de la Sra. $\mathrm{X}$ y lo devolvió a su madre" quien, en aquel momento, residía en una barraca en una zona deprimida de la ciudad. Allí conoció a una pareja a quien entregó informalmente al niño para que lo cuidaran. Estos presentaron una solicitud legal de guarda y criaron al niño durante 4 años, hasta que se revelaron los malos tratos que le infligían. Tras serles retirada la guarda, el niño fue devuelto a su madre biológica, quien, por su cuenta, volvió a entregarlo a otra familia. Tras una semana con él, lo devolvieron de nuevo a su madre. El informe expone que el niño padeció malos tratos tanto por parte de esta como de su padrastro, por lo que se supendió la patria potestad a su padre, el menor fue declarado adoptable y pasó a una acogida -formal, por primera vez- en un orfanato. Allí se propuso su adopción a una mujer con quien convivió durante tres años; sin embargo, pasado este tiempo, la madre adoptiva renunició alegando no estar preparada para la adopción. En aquel momento el menor tenía ya siete años; dos años después fue asignado a una mujer (adopción monoparental) que renunció al cabo de un año por problemas de conducta y mal comportamiento.

Obviamente, la edad y trayectoria de los padres/madres adoptivos es un factor de riesgo relevante. Por ejemplo, la madre de Olga, nacida en un país del este de Europa, fue abandonada siendo menor de edad y pasó a ser acogida por una pareja. Se quedó embarazada de la niña y tras el nacimiento de esta se fue definitivamente del hogar de acogida, dejando a la pequeña a cargo de los "abuelos" acogedores, quienes renunciaron a su tutela cinco años después. Desde el ingreso en el sistema gubernamental de protección, Olga estuvo a cargo de otras dos familias, fracasando el acogimiento en ambos casos. Finalmente, fue adoptada en Cataluña un año más tarde por una mujer (adopción monoparental), quien solo un mes después acudió al ICAA manifestando la voluntad de renunciar a la adopción puesto que la niña era "muy desobediente" y se veía obligada a castigarla "porque no hacía caso". Para reforzar su decisión esgrimió que la niña ya había sido rechazada por dos familias anteriormente "porque era muy difícil" y que se le habían dado a ella "porque era mayor y soltera".

\section{Conclusión}

Los factores que inciden en el bienestar del menor durante su periodo de crianza son diversos y pueden confluir desde múltiples dimensiones simultáneamente (factores laborales, económicos, ambientales, sanitarios, etc.). Hay ocasiones en que, por diversas razones, el cuidado familiar se interrumpe y el menor requiere la intervención de otras personas o instituciones que asuman la responsabilidad última de su cuidado. La fórmula jurídica requiere una declaración legal de desamparo, emitida por la autoridad judicial competente. Aunque no siempre es la única vía de resolución, por cuanto los emplazamientos informales (circulación) al margen de los canales jurídicos son una fórmula frecuente en diversos países de origen de los menores adoptados internacionalmente en España. Nuestro estudio explora la adversidad que sobreviene una vez el NNA ha ingresado en el sistema formal de protección y se encuentra ante nuevas situaciones de desatención o desamparo.

Esta recursividad en algunos casos (pocos, si los comparamos con el conjunto de menores amparados por el sistema de protección, pero cuyas consecuencias pueden ser devastadoras para los NNA implicados) nos enfrenta a las cadenas de vulnerabilidad no resueltas institucionalmente y cuya sucesión agrava irremediablemente el bienestar del menor y puede abocarle a un círculo vicioso de reinstitucionalización y desamparo que puede condicionar su personalidad y equilibrio emocional en el futuro (Meakings y Selwyn 2016, Brännström y otros 2020, Eldridge y otros 2020). En esta línea, nuestro estudio se adentra en las conexiones entre acogimientos familiares fallidos y truncamientos adoptivos, contribuyendo a rellenar los relativos vacíos de información señalados por Sattler y Font (2020) o Brodzinsky y Smith (2018), incorporando las situciones de circulación informal de menores que ya detectaban estos últimos autores. Para ello, hemos conseguido acceder a la totalidad de información 
oficial registrada para los casos de truncamiento adoptivo en Cataluña, superando las constricciones señaladas por Dellor y Freisthler (2018) y aportando información sobre factores que pueden influir en el proceso.

Así, adicionalmente a los hallazgos de Berástegui (2003) en la comunidad de Madrid y de Paniagua y Palacios (2018) en Andalucía, respecto a la baja incidencia aculmulada y a factores de riesgo vinculados a la edad del menor o a su comportamiento, con quienes coincidimos, nuestro análisis de los casos permite concluir lo siguiente:

a) Tanto la circulación informal como el acogimiento legal pueden llegar a dañar gravemente la integridad de un/a menor. Tratándose en ambos casos de medidas que pretenden paliar situaciones de vulnerabilidad, en ocasiones las personas que asumen la crianza de estos niños y niñas incumplen las obligaciones implícitas en dicha crianza.

b) Pueden hallarse experiencias similares de circulación o acogimiento informal, de acogimiento legal y también de fracasos de ambas medidas en países muy distintos desde el punto de vista político y cultural.

c) En no pocos casos el menor es reiteradamente emplazado, de forma informal, a causa, sobre todo, de dificultades económicas o por la detección de los fracasos en los acogimientos.

d) Como ya han reclamado insistentemente investigaciones precedentes en el ámbito del acogimiento y la adopción (Twigg y Swan 2007, Schwartz 2008), es insuficiente el establecimiento de estas medidas por parte de la administración si no se robustece el apoyo a las familias que acogen a NNA. En procedimientos de adopción internacional subsiguientes a procesos de acogimiento familiar previo, es importante que este acompañamiento se haya dado tanto en origen como en destino. Tras la detección de cualquier dificultad en los procesos de adaptación, debe incrementarse la proactividad en el seguimiento y apoyo. Todo ello, como apuntan numerosos estudios precedentes (por ejemplo, Fernández del Valle y otros 2009, y López y otros 2014), requiere por parte de las instituciones públicas destinadas a garantizar la protección del menor de una inversión económica y en equipo humano que permita cumplir adecuadamente su función.

Por último, debemos señalar una limitación evidente del estudio: las considerables consecuencias de la pandemia sobre el trabajo de campo. Futuras investigaciones, que puedan produndizar en este ámbito bajo contextos más propicios, deberían aumentar el número de informantes (tanto en lo referente a jóvenes extutelados y a familias de acogida fallida, como a representantes de la propia administración).

\section{Notas}

Este artículo recoge algunos resultados del proyecto de investigación Crianza, desamparo y vulnerabilidad sociocultural. Análisis situacional y propuestas de intervención(CSO2017-83101-C2-1-R), codirigido por el Dr. Jorge Grau Rebollo y la Dra. Anna Piella Vila y financiado por el Ministerio de Ciencia, Innovación y Universidades.

1. Por ejemplo, Fernández-Daza (2018: 272) señala, en el ámbito iberoamericano, cómo al acogimiento familiar se le denomina: acogimiento familiar; familia de acogida o de acogimiento, familias sustitutas, familias acogedoras, familias cuidadoras, hogares de acogimiento familiar, hogares sustitutos, hogares solidarios, familias solidarias, medida o colocación en medio familiar, o familia amiga.

2. Con posterioridad a la Convención sobre los Derechos del Niño de 20 de noviembre de 1989 impulsada por Naciones Unidas, España desarrollará la Ley Orgánica 1/1996, de 15 de enero, de Protección Jurídica del Menor y la Ley 26/2015, de 28 de julio, de modificación del sistema de protección a la infancia y a la adolescencia. 
Allueva, Laura

2011 "Situacions de risc i desemparament en la protecció de menors", Indret: Revista para el análisis del Derecho, $n^{\circ} 4: 1-24$.

Berástegui, Ana

2003 Las adopciones internacionales truncadas y en riesgo en la comunidad de madrid. Madrid, Consejo Económico y Social (Comunidad de Madrid).

2018 "Variables en la definición de los fracasos en la adopción, variables en el éxito de las adopciones", en C. Jeannin (ed.), Fortaleciendo las competencias: Aprender de los fracasos en la adopción internacional, Ginebra, Servicio social internacional, Centro internacional de referencia para los derechos del ninño privado de familia: 19-22.

Boletín Oficial del Estado

2015 Ley 26/2015, de 28 de Julio, de modificación del sistema de protección a la infancia y a la adolescencia.Madrid, Jefatura del Estado. https://www.boe.es/buscar/act.php? ?id=BOE-A-2015-8470

Brady, Ivan (ed.)

1976 Transactions in kinship : adoption and fosterage in Oceania. Honolulu, University Press of Hawaii.

Brännström, Lars (y otros)

2020 "Outcomes in adulthood after long-term foster care: a sibling approach", Child maltreatment, vol. 25 n4: 383-392.

Brodzinsky, David (y Susan Livingston Smith)

2018 "Commentary: understanding research, policy, and practice issues in adoption instability", Research on social work practice, vol. $29, \mathrm{n}^{\circ} 2$ : 185-194.

Casas, Ferran

2012 La protecció dels infants $i$ adolescents en situació de risc social i desemparament a Catalunya. Barcelona. http://www.tiac.cat/images/lloc/comissio/DOCS-PROTECCIO_INFANCIA.pdf

Chanyalew, Sophia (y Tatek Abebe)

2016 "Qenja: child fostering and relocation practices in the Amhara region, Ethiopia", Children's geographies, vol. 14, $\mathrm{n}^{\circ}$ 1: 46-62.

Colbridge, Alicia K. (y otros)

2017 "'Who am I?' How female care leavers construct and make sense of their identity", SAGE Open, vol.

7. DOI: https://doi.org/10.1177/2158244016684913.

https://journals.sagepub.com/doi/pdf/10.1177/2158244016684913

Colton, Mathrew (y Margaret Williams)

1997 "The nature of foster care internacional trends", Adoption \& Fostering vol. 21: 44-49.

Dávila, Paulí (y Luis Naya)

2006 "La evolución de los derechos de la infancia: una visión internacional", Encounters on Education, vol. 7: 71-93.

Dellor, Elinam (y Bridget Freisthler)

2018 "Predicting adoption dissolutions for children involved in the child welfare system", Journal of child custody vol. 15: 136-146. https://doi.org/10.1080/15379418.2018.1460001.

DGAIA

2019 Informe estadístic mensual. Barcelona, Direcció general d'atenció a la infància i l'adolescència. https://treballiaferssocials.gencat.cat/web/.content/03ambits_tematics/07infanciaiadolescencia/dades sistema_proteccio/2019_01_informe_DGAIA.pdf

Eldridge, Joshua (y otros)

2020 "Confiding in others: exploring the experiences of young people who have been in care", Adoption \& Fostering, vol. 44: 156-172.

Fernández-Daza, María Patricia

2018 "El acogimiento familiar en Iberoamérica", Saude soc. vol. 27: 1-11. 
Fernández del Valle, Jorge (y otros)

2009 "El acogimiento familiar en España: implantación y retos actuales", Papeles del psicólogo, vol. 30: $33-41$.

Fernández del Valle, Jorge (y otros)

2008 El acogimiento familiar en España. Una evaluación de resultados. Madrid, Ministerio de Trabajo y Asuntos Sociales. Subdirección General de Información Administrativa y Publicaciones.

Festinger, Trudy

2002 “After adoption: dissolution or permanence?", Child Welfare, vol. 81: 515-533.

Festinger, Trudy (y Penelope Maza)

2009 "Displacement or post-adoption placement? A research note", Journal of Public Child Welfare, vol. 3: $275-286$.

Fonseca, Claudia

2002 "Inequality near and far: adoption as seen from the Brazilian favelas", Law and Society Review, vol. 36: 397-431.

2004 "Pautas de maternidad compartida entre grupos populares de Brasil", en Diana Marre (y Joan Bestard) (ed.), La adopción y el acogimiento. Presente y perspectivas. Barcelona, Publicacions i Edicions de la Universitat de Barcelona: 91-116.

2006a "Da circulação de crianças à adoção internacional: questões de pertencimento e posse", Cadernos Pagu, vol. 26: 11-44.

2006b "Transnational influences in the social production of adoptable children: the case of Brazil", International Journal of Sociology and Social Policy, vol. 26: 154-171.

García Tugas, Lourdes

2019 La cara oculta de la adopción. Las adopciones internacionales truncadas en Cataluña. Tesis doctoral. Departamento de Antropología Social y Cultural, Universitat Autònoma de Barcelona. https://www.educacion.gob.es/teseo/imprimirFicheroTesis.do

Generalitat de Catalunya

2010 "Ley 14/2010, de 27 de mayo, de los derechos y las oportunidades en la infancia y la adolescencia", Boletín Oficial Del Estado, nº 156 (10213): 56372-56433.

Goody, Jack

1969 "Adoption in cross-cultural perspective", Comparative studies in society and History, vol. 11: 55-78.

Goody, Jack (y Esther Goody)

1967 "The circulation of women and children in Northern Ghana", Man, vol. 2: 226-248.

Grau Rebollo, Jorge

2011 "Parentesco, adscripción y crianza. Elaboraciones culturales de la adopción internacional y la circulación de niños", Revista de Antropología Social, n² 20: 31-54.

Grotevant, Harold (y otros)

2000 "Adoptive identity: how contexts within and beyond the family shape developmental pathways", Family Relations, vol. 49: 379-387.

Howard, Neil

2011 "Is 'child placement' trafficking? Questioning the validity of an accepted discourse", Anthropology Today, vol. 27: 3-7.

Howell, Signe

2009 "Adoption of the unrelated child: some challenges to the anthropological study of kinship", Annual Review of Anthropology, vol. 38: 149-166.

Kim, JaeRan

2017 "'You can't run into a burning building without getting burned yourself': An ecological systems perspective of parents choosing out-of-home care for an intercountry adopted child", Families in Society, vol. 98: 169-77. https://doi.org/10.1606\%2F1044-3894.2017.98.28. 
Kim, JaeRan (y otros)

2019 "Adoption discontinuity in intensive out-of-home care settings", Adoption Quarterly, vol. 22: 307-329. https://doi.org/10.1080/10926755.2019.1675838.

Leinaweaver, Jessaca

2007 "On moving children: the social implications of Andean child circulation", American Ethnologist, vol. 34: 163-180.

2008 The circulation of children: kinship, adoption, and morality in Andean Peru. Durham, Duke University Press.

2010 "Outsourcing care: how peruvian migrants meet transnational family obligations", Latin American Perspectives, vol. 37: 67-87.Li, Dongdong (y otros)

2017 "Comparing Long-term placement outcomes of residential and family foster care: a meta-analysis", Trauma, Violence \& Abuse, vol. 20: 653-664.

López, Mónica (y otros)

2014 "Características y desarrollo del acogimiento familiar en dos países con fuerte tradición de acogimiento residencial: España y Portugal”, Universitas Psychologica, vol. 13: 865-880.

Marre, Diana (y Laura Briggs) (ed.)

2009 International adoption: global inequalities and the circulation of children. New York, New York University Press.

Martín, María

2020 "España no sabe cuántos menores extranjeros no acompañados acoge", El País (edición digital, Madrid), 14 de mayo. https://elpais.com/espana/2020-05-13/espana-no-sabe-cuantos-menoresextranjeros-no-acompanados-acoge.html

McCall, Robert

2011 "Research, practice, and policy perspectives on issues of children without permanent parental care", Monographs of the society for research in child development, vol. 76: 233-272. DOI: https://dx.doi.org /10.1111\%2Fj.1540-5834.2011.00634.x.

Meakings, Sarah (y Julie Selwyn)

2016 "'She was a foster mother who said she didn't give cuddles': the adverse early foster care experiences of children who later struggle with adoptive family life", Clinical child Psychology and Psychiatry, vol. 21: 509-519. https://doi.org/10.1177\%2F1359104516631608.

Observatorio de la Infancia

2020 Boletín de datos estadísticos de medidas de protección a la infancia. Madrid, Observatorio de la Infancia.

Palacios, Jesús (y David Brodzinsky)

2010 "Review: Adoption research: trends, topics, outcomes", International Journal of Behavioral Development, vol. 34: 270-284. DOI: 10.1177/0165025410362837

Palacios, Jesús (y otros)

2009 "Intercountry adoption disruptions in Spain", Adoption Quaterly, vol. 9: 35-55.

https://doi.org/10.1300/J145v09n01_03.

Paniagua, Carmen (y Jesús Palacios)

2016 "Adopciones rotas en Andalucía: caracterización y propuestas para la intervención", Apuntes de Psicología, vol. 34: 301-309.

Paniagua, Carmen (y otros)

2018 "Adoption breakdown in Spain: A survival and age-related analysis", Research on social work practice, vol. 29: 176-84. https://doi.org/10.1177\%2F1049731518791037.

Real, Marta (y otros)

2020 "Acogimiento familiar en España: un estudio de revisión”, Aposta. Revista de ciencias sociales, vol. 84: 8-24. 
Rollet, Catherine

2001 "La santé et la protection de l'enfant vues a travers les congrès internationaux", Annales de démographie historique, vol. 101: 97-116.

Rolock, Nancy

2015 "Post-permanency continuity: what happens after adoption and guardianship from foster care?", Journal of public child welfare, vol. 9: 153-173. https://doi.org/10.1080/15548732.2015.1021986.

Rolock, Nancy (y otros)

2018 "A comparison of foster care reentry after adoption in two large U.S. states", Research on Social Work practice, vol. 29: 153-164. https://doi.org/10.1177\%2F1049731518783857.

Sattler, Kierra (y Sarah Font)

2020 "Predictors of adoption and guardianship dissolution: the role of race, age, and gender among children in foster care", Child Maltreatment, vol. 26: 1-12. https://doi.org/10.1177\%2F1077559520952171.

Schwartz, Lita Linzer

2008 "Foster care and social services", The journal of Psychiatry \& Law, vol. 36: 609-615. https://doi.org /10.1177\%2F009318530803600405.

Twigg, Robert (y Tracy Swan)

2007 "Inside the foster family: what research tells us about the experience of foster carers' children", Adoption \& Fostering, vol. 31: 49-61. https://doi.org/10.1177\%2F030857590703100407.

\section{UNICEF}

2006 Convención sobre los derechos del niño. Madrid, UNICEF Comité Español.

https://www.unicef.es/publicacion/convencion-sobre-los-derechos-del-nino

West, Delphine (y otros)

2020 "Attachment in family foster care: literature review of associated characteristics", Developmental child welfare, vol. 2: 132-150. https://doi.org/10.1177\%2F2516103220915624.

Wolf, Klaus

2008 "Foster care research in Germany: A critical review", Adoption \& Fostering, vol. 32: 19-30. https://doi.org/10.1177\%2F030857590803200204.

Zwimpfer, Diane

1983 "Indicators of adoption breakdown", Social Casework, vol. 64: 169-177.

https://doi.org/10.1177\%2F104438948306400306. 